\title{
FAILURE OF MYOCARDIAL TISSUE: SIMULATION OF BLOOD PERFUSION
}

\author{
SUMESH SASIDHARAN ${ }^{1}$, PETER H.M.BOVENDEERD² AND JACQUES \\ M.HUYGHE ${ }^{1,2}$
}

\author{
${ }^{1}$ Bernal Institute \\ University of Limerick, Ireland \\ sumesh.sasidharan@ul.ie
}

${ }^{2}$ Department of Biomedical Engineering

Eindhoven University of Technology,Netherlands

p.h.m.bovendeerd@tue.nl, jacques.huyghe@ul.ie

Key words: Coronary Blood Perfusion, Cardiac Modelling, Poromechanics.

\begin{abstract}
Technological advances in computational mechanics allow to simulate failure of any part of airplanes, pumps, compressors, electric power stations, foundations, bridges, etc. Billions of dollars have been invested to calculate 3D stress configuration in engineering structures. Because the criterion of failure in engineering structures is generally exceeds stress. Failure of the human heart is responsible for 6 million deaths per year worldwide.

Models of cardiac mechanics have been developed to analyse cardiac pump function from tissue to organ scale. All of these models focus on stress and strain. However, heart failure is not associated with fracture. Failure of a heart is usually induced by a mismatch between blood perfusion and metabolic needs of the cardiomyocytes. Because failure is associated with blood perfusion and present day models do not address this failure mechanism, there is an urgent need for a computational strategy for blood perfusion in deforming myocardial tissue. Upscaling of the vessel trees to a continuum opens the way to computation of coronary blood flow in a multi compartment poro-mechanical model of the beating heart. Arterial, arteriolar, capillary, venular and venous blood are treated as separate compartments. As a result, the supply of oxygen to the tissue is modelled. The present interest in tissue engineering as means to support heart function, and the great difficulties associated with angiogenesis in myocardial tissue engineering, calls for a virtual environment for testing cardiac interventions, so that the time to market of newly designed devices and therapies can be shortened substantially.
\end{abstract}

\section{INTRODUCTION}

Engineers designing any part of a motor know that stress is a precursor of failure. Therefore, they have at their disposal the most advanced and user-friendly Computer Aided Engineering software for computing stress in any minute part of any engineering design. This explains the high reliability of engineering designs today. Unlike engineering 
designs, the human heart does not fail because of excess stress. It fails because of lack of regional coronary blood perfusion in parts of the cardiac muscle leading to various coronary vascular diseases(CVD).Despite dramatic medical advances over the last few decades, CVD remain a leading cause of death globally and the number one cause of death in the world [1]. While present day finite element(FE)models compute stress and strain in the heart muscle [2],they do not compute the regional blood perfusion and therefore missthe key factor that may lead to cell necrosis and failure of the heart.

Coronary vascular disease (Fig 1) has been studied for many decades and has been found to be a complex problem. Despite the tedious and creative work by many expert scientists from many different perspectives [1,2]the disease has only partially been understood. The current state of the art in cardiac FE modelling provides the capability to compute myocardial stresses and strains $[3,4]$ and has led to successful commercialisation of the code for simulation of the ability of cardiac devices [5] to improve the performance ofthe heart in different pathologies. Studies [6] have also demonstrated that systole inhibit coronary blood flow by a waterfall mechanism. Despite the importance of coronary vascular perfusion, FE modelling of cardiac tissue has greatly overlooked "perfusion" as a key component of cardiac health. Some studies have attempted to fill this gap by means of network analyses of vascular perfusion on minute portions of a microvascular network [7]. The studies[8] on fractal nature have shown that fractal nature of spatial flow distribution in the heart, and of temporal intravascular washout, are explicable in terms of the morphometry of the coronary network. The review presented in [9] describes why coronary flow is Vankan et al. [19] con model and an explici computer-generated hierarchical porous soli
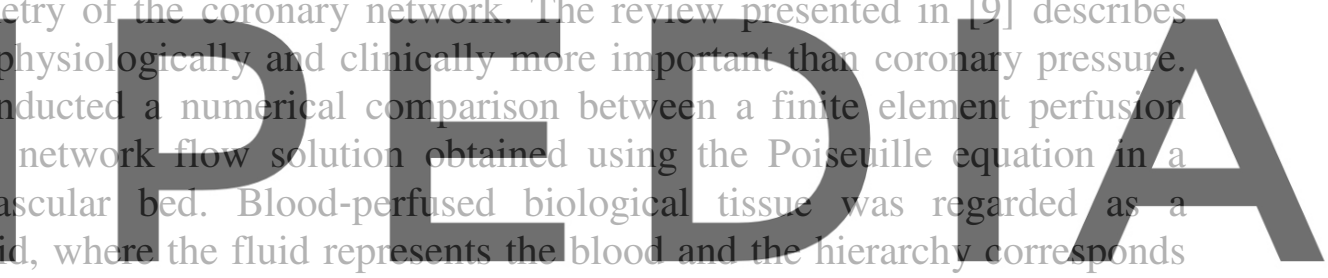

to the tree-like vascular structure. The approach that is taken here differs in basic tenets from

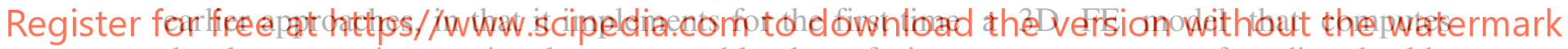
the key quantity: regional coronary blood perfusion as a measure of cardiac health. Even though perfusion simulation are possible with finite element such methods have never been used for coronary circulation and the proposed work will fill the gap and advance the state of the art by opening the gateway to detailed numerical modelling of the full mechanobiology of this vital organ including oxygen delivery, $\mathrm{CO}_{2}$ draining, autoregulation, balance between energy expenditure and supply [10]. Fractional flow reserve [11] has been used in coronary catheterization across a coronary artery stenosis to determine the likelihood that the stenosis impedes oxygen delivery to the heart muscle. A well validated model of the myocardial contraction and perfusion of the patient's heart,based on hemodynamic data collected by the cardiologist, may improve the quantification of the fractional flow reserve of the patient and hence the decision of the cardiologist to go forward with stent surgery or conservative treatment.

Objective 1 Cardiac Modelling. The Fig. 2 shows the 3D FE model as used for stress and deformation analysis of a full heartbeat. No coronary perfusion has been included into the model yet. Colour coded plots of this is being produced for venous, venular, capillary, arteriolar and arterial pressure, regional coronary perfusion as well as arrow plots of corresponding flow. Lumped parameter approaches have been successful in 
modelling the overall conductance and capacitance of the coronary system. The earliest resistance-compliance element windkessel, lumped parameter model for the circulation system was developed during beginning of 1900. Lumped parameter models can only predict blood flows in the main systematic arteries, however, do not integrate the actual structure of the microvasculature [12,13]. The continuum approach as proposed by authors does include microstructure into the model while abstaining from accounting for each vessel separately. While the micro-macro transformation has been completely worked out including a formal verification to real world examples [14], this transformation has never been applied to the field most relevant to cardiac health issues, i.e. coronary circulation. This intrinsic multiscale approach has been successfully integrated into FE models of skeletal muscle. Figure 2 shows a comparison of pre-capillary pressures computed by means of network analysis and compared to continuum(FE) analysis.

Objective 2: Coronary perfusion. It is known that vast majority of the life threatening pathologies are associated with an imbalance between the demand in oxygen and the supply provided by the RBCs. Microvascular network analysis works fine for a few hundred micro vessels, and sheds light on the microvascular mechanisms regulating flow. However, this approach is doomed to failure when it comes to perfusion studies of the heart as a whole as there are 3,000 capillaries per square millimetre in the human heart. This project will start studying the structure of the microvascular geometry and upscale the coronary circulation from there. The microvascular bed will be embedded into a beating heart with all complexities associated with it: contracting muscle fibre, complex fibre architecture, valves, the arterial coronary injected post mortem after polymerisation The cast shows the arteric bifurcate from the large aortic arch: one on the right, the other on the left. The artery seen on

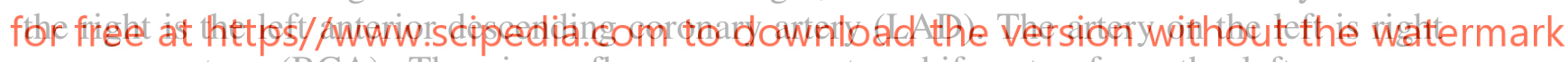
coronary artery (RCA). The circumflex coronary artery bifurcates from the left coronary artery. Entangled with the arterial tree is an equally complex venous coronary tree that drains the blood from the myocardium back to the vena cava. Figure 3(b) shows the left and right ventricles filled with white polymer with the blue vessels showing the largest coronary veins, that drains vessels of the heart. Using low viscosity monomer, the smallest vessels can be visualised as shown in Figure 4. The capillaries ( 5 micron in diameter) that run parallel to the muscle fibers, spring from the arterioles and drain the blood to the venules.

Methodology : The key quantity of this proposal is coronary blood perfusion. It is defined as the volume of blood flowing through the capillaries of a unit volume of tissue per unit time. Its dimension is 1/time. Typical value at rest is $0.01 \mathrm{~Hz}$. Unlike any existing lumped parameter model of coronary perfusion, the parameters of the model will be measured directly from the coronary vascular tree of postmortem human hearts using the formula: $K_{i j}=\frac{n^{b}}{32}<\frac{d^{z}}{\mu(d)} \frac{\Delta x_{i}}{\Delta s} \frac{\Delta x_{j}}{\Delta s}>i, j=0,1,2,3$. where $\mathrm{K}_{\mathrm{ij}}$ is the conductance matrix of the compartment, $\mathrm{n}^{\mathrm{b}}$ is its volume fraction, angle brackets denote an averaging over a representative volume, $d$ is the diameter of the vessel segment, $\mu(d)$ is its apparent viscosity and $\left(\Delta x_{i}\right) / \Delta s$ its direction cosines in the spatial and arteriovenous directions. Figure 5 shows 
the computer generated arterial vascular tree mode. While the micro-macro transformation has been completely worked out including a formal verification to real world examples, this transformation has never been applied to the field most relevant to public health issues, namely the coronary circulation. These trees will include all vessels from veins, venules, capillaries, arterioles and arteries [15]. The numerical treatment of the differential equations will be using the mixed hybrid finite element method [16,17,18] ensuring local mass balance at all times. The Raviart-Thomas element with 8 corner nodes with 3 nodal displacements is used. The fluxes through the six sides of the hexahedron have one flux degree of freedom per microvascular compartment. The number of compartments can be set according to findings. If they are three in number, one arteriolar, capillary flux and venular flux, 3 Lagrange multipliers per side is introduced to enforce local mass continuity for each of the three microvascular compartments. The fluxes are eliminated to the profit of the Lagrange multipliers. The 3D finite element code describing the finite deformation of a depolarising beating heart has been developed by authors and extended to include coronary blood perfusion. Mixed hybrid finite element approach, appears to be far superior to the classical u-p formulations typically used by commercial codes for poromechanics, such as ABAQUS or ANSYS. This is particularly important in the case of coronary perfusion because it deals with a strongly heterogenous and anisotropic medium which will be subjected to heterogenous boundary conditions in the case of coronary artery occlusion.

The myocardial space is subdivided in a solid volume fraction and a fluid volume fraction which in turn is subdivided in a spectrum of compartments. The flux of the coronary blood is represented as a 4D vector in the space spanned by the 3 dimensions of the myocardium and the arteriovenous dimension. The extended Darcy equation the fluxes to the computed from the vessel geometries and substituted into each area of the myodardial wal microvascular compartment.

Because

the conductance matrix is computed from

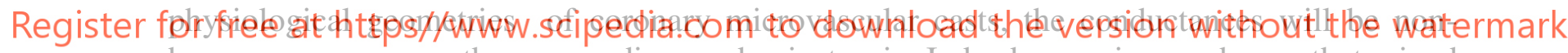
homogeneous across the myocardium and anisotropic. Indeed experience shows that mixed hybrid methods perform better than conforming elements in strongly non-homogeneous media. A single fluid compartment would be built into the existing finite deformation model of the beating heart using mixed hybrid finite elements and(ii) then implement the multiple fluid compartment model of a non-deforming compliant microvasculature. Both models will be tuned separately and integrated to experimental data from the literature [19-21]

$$
\begin{gathered}
\frac{\partial v}{\partial t}+\nabla \cdot q=0 \\
q=-K \nabla p \\
p=p_{I M}+p_{T M}
\end{gathered}
$$

$\mathrm{v}=$ volume fraction of blood compartment 


\section{$\nabla$ : four dimensional gradient operator.}

$\mathrm{q}=$ specific volume of blood in compartment

$\mathrm{K}=$ conductance matrix

$\mathrm{p}=$ blood pressure of compartment

$\mathrm{p}_{\mathrm{IM}}=$ intramyocardial pressure :

$\mathrm{p}_{\mathrm{TM}}=$ Transmural pressure

Substitute (2) in (1)

$$
\nabla \cdot K \nabla p=\frac{\partial v}{\partial t}
$$

With boundary conditions

$$
p\left(X_{0}^{a}\right)=P_{E P I}^{\text {art }} \quad \text { and } p\left(X_{0}^{v}\right)=P_{E P I}^{\text {vein }}
$$

Along the epicardial surface, $P_{E P I}^{\text {art }}$ is the arterial prescribed blood pressure

$P_{E P I}^{\text {vein }}$ is the venous prescribed blood pressure

Eq (4) holds within the 4

arteriovenous parameters

All other surfaces of $\Omega$ have the
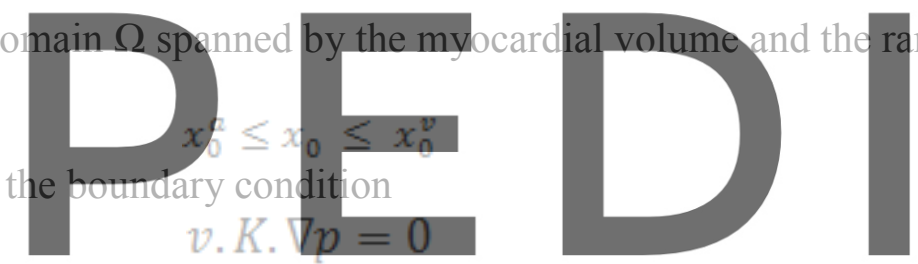

(5)

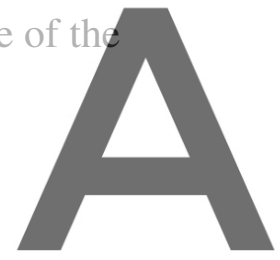

Register for free at https//www.scipedia.com to download the version without the watermark

We define a weighting function $\psi$ and transform eq (1) into a weighted residual formulation

$$
-\int_{\Omega} \psi \nabla \cdot q \cdot d \Omega=\int_{\Omega} \psi \frac{\partial u}{\partial t} d \Omega
$$

We define a vectorial trial function $\mathrm{V}$ and transform eq (2) into a weighted residual formulation.

$\mathrm{C}=\mathrm{K}^{-1} \quad$ Hence $\nabla p=-C q$

$$
\begin{gathered}
\int_{\Omega} V \cdot C \cdot q \cdot d \Omega+\int_{\Omega} V \cdot \nabla p d \Omega=0 \\
\int_{\Omega} V \cdot C \cdot q \cdot d \Omega-\int_{\Omega} p \nabla \cdot V d \Omega=
\end{gathered}
$$




$$
\int_{X_{0}^{a}}^{a} p_{E P I}^{a r t} v \cdot V d s-\int_{\Theta p i} p_{E P I}^{v e i n} v \cdot V d s(7)
$$

We define a Raviant Thomas finite element mesh in the domain $\Omega$. Within each element, we interpolate the fluxes, $\mathrm{q}$ with the I interpolation function $\mathrm{V}_{\mathrm{i}}$

$$
q=\sum_{i=1}^{I} q_{i} V_{i}
$$

While the pressure $\mathrm{p}$ is interpolated with the $\mathrm{J}$ interpolation function $\psi_{j}$

$$
p=\sum_{j=1}^{J} p_{i} \psi_{j}
$$

Eqs (8) and (9) are substituted in eqs (6) and (7)

Using Galerkin method we choose weighing functions identical to interpolation functions

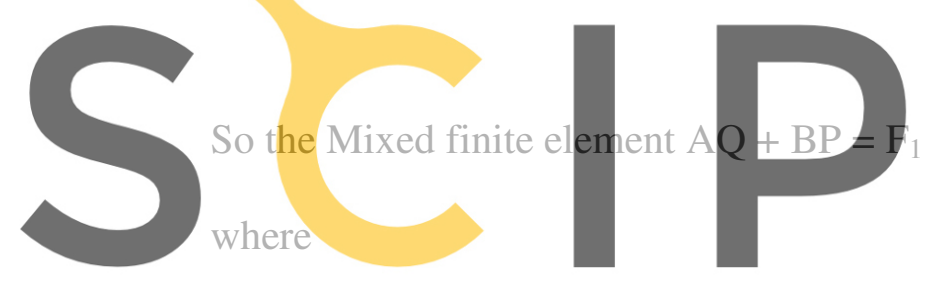

$$
\mathrm{V}=\mathrm{V}_{\mathrm{j}}
$$

Register for free at https//www.scipedia.com to downtoad the version without the watermark
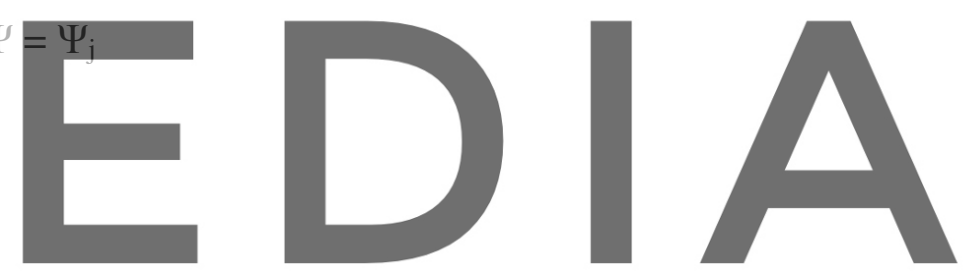

$$
\begin{aligned}
& B=-\int_{\Omega} \psi_{j} \cdot \nabla v_{i} \cdot d \Omega . \\
& \mathrm{B}^{\mathrm{T}} \mathrm{Q}+\mathrm{DP}=-\mathrm{F}_{2}
\end{aligned}
$$

Time dependant behaviour due to elasticity of the vessel wall.

At time, $\mathrm{t}=\mathrm{t}_{\mathrm{n}-1}$, we know the values of $\mathrm{Q}$ and $\mathrm{P}: \mathrm{Q}_{\mathrm{n}-1}$ and $\mathrm{P}_{\mathrm{n}-1}$

At time $t=t_{n}$, we do not know the values of $Q$ and $P$ : $Q_{n}$ and $P_{n}$

So for the MHFE, we have $\Lambda$ - Lagrangian multiplier (to make flux coming out of one element same as flux going into next element)

$$
\mathrm{AQ}+\mathrm{BP}+\mathrm{C} \Lambda=\mathrm{F}_{1}
$$




$$
\begin{aligned}
& \mathrm{B}^{\mathrm{T}} \mathrm{Q}+\mathrm{DP}=-\mathrm{F}_{2} \\
& \mathrm{C}^{\mathrm{T}} \mathrm{U}=\mathrm{F}_{3}
\end{aligned}
$$

Eliminating $\mathrm{Q}$ and doing time integration for $\mathrm{P}$ will give the final equation $\mathrm{E} \Lambda=\mathrm{F}$

\section{Results and Discussion}

The figure 6 shows preliminary results of the implementation of Mixed Hybrid Finite element where the model working for potential rigid porous models in $2 \mathrm{D}$ and $3 \mathrm{D}$. The hydraulic permeability chosen for the models is a unit matrix. The examples presented here have it for a flow outside the in 2D (Fig 6 a) and flow inside 3D models (Fig 6b ). It will be extended to axi-symmetric and full 3D models

\section{Conclusions}

The presented study provide earlier insights on the successful implementation of mixed hybird finite element model for use in coronary perfusion studies There are far-reaching implications of developing a cardiac model with coronary perfusion. The model opens the gateway to detailed numerical modelling of the full mechanobiology of this vital organ including oxygen delivery, CO2draining, autoregulation, balance between energy expenditure and supply. Although implementing the mechanobiologyof the myocardial tissue is far beyond the capabilities of this modest project, we anticipate that this work will create this possibility for further projects. The scope of the study is usage of FE modelling for autoregulation of coronary perfusion which extends the applications of FE model to common pathologies such as ischemic as stents or cardiac as

\section{REFERENCES}
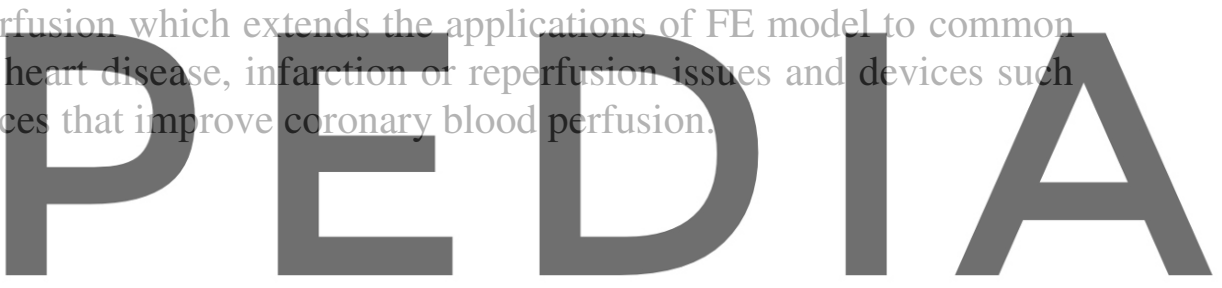

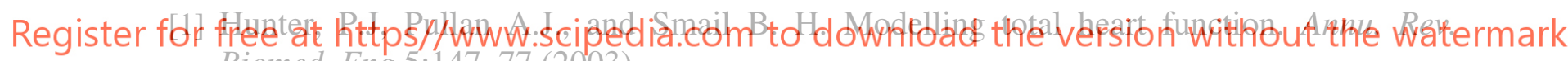
Biomed. Eng.5:147-77 (2003)

[2] Weese J., Smith N., Ravazi R., Chapelle D., Delingette, H., Frangi A., Hose R., Hunter P.

Spaan J.EUHEART-project: Personalised \& integrated cardiac care: patient specific cardiovascular modelling and simulation for in silico disease understanding \& management and for medical device evaluation\& optimisation. FP7-ICT-2007. Final report (2013).

[3] Pluijmert, M.H., Bovendeerd, P.H.M., Kroon, W., Prinzen, F. \& Delhaas, T. Effects of activation pattern and active stress development on Myocardial shear in a model with adaptive myofiber reorientation. Am. J. of Physiol : Heart Circ. Physiol., 306, H538H546 (2014).

[4] Bovendeerd, P.H.M. Modeling of cardiac growth and remodeling of myofiber orientation. Journal of Biomechanics, 45(5), 872-881(2012)

[5] Ramesh Marrey, Brian Baillargeon, Maureen L. Dreher, Jason D. Weaver, Srinidhi Nagaraja, Nuno Rebelo, Xiao-Yan Gong. Validating Fatigue Safety Factor Calculation Methods for Cardiovascular StentsJ Biomech Eng, Jun 1;140(6)(2018)

[6] Calvin Eng and Edward S. Kirk Flow into Ischemic Myocardium and across CoronaryCollateral Vessels is Modulated by a Waterfall Mechanism Cir Res 10-17 
(1984).

[7] Popel A.S., Johnson P.C. Microcirculation and Hemorheology. Annu. Rev. Fluid Mech. 37: 43-69 (2005)

[8] Daniel A. Beard James B. Bassingthwaighte The Fractal Nature of Myocardial BloodFlow Emerges from a Whole-Organ Model of Arterial Network J.Vasc Res 37:282296 (2000)

[9] Tim P. van de Hoef, Maria Siebes, Jos A. E. Spaan, Jan J. Piek, Fundamentals in clinical coronary physiology: why coronary flow is more important than coronary pressure European Heart Journal, 36(47), 3312-3319. (2015)

[10] Jianlong Kou, Yanyan Chen, Xiaoyan Zhou, Hangjun Lu, Fengmin Wu, Jintu Fan Optimal structure of tree-like branching networks for fluid flow PhysicaA 393 527-534 (2014).

[11] Ioannis P. and D. Katritsis, Fractional Flow Reserve Derived from Coronary Imagingand Computational Fluid Dynamics Interv Cardiol. 9(3): 145-150 (2014)

[12] Westerhof, N., Bosman, F., De Vries, C. J. \& Noordergraaf, A. Analog studies of the human systemic arterial tree. Journal of biomechanics 2, 121-143 (1969)

[13] Huyghe J.M., Oomens C.W., Campen D.H. van, Heethaar R.M. Low Reynolds number steady flow through a branching network of rigid vessels: I. A mixture theory. Biorheology 26, 55-71 (1989).

[14] Huyghe J.M., Oomens C.W., Campen D.H. van. Low Reynolds number steady state flow through a branching network of rigid vessels: II. A finite element mixture model

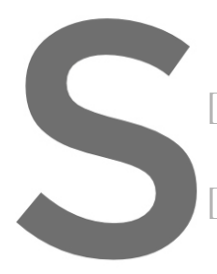
Biorheology 26,

[15] Huyghe J.M., porous solids: balat

6] Huyghe J.M., Can
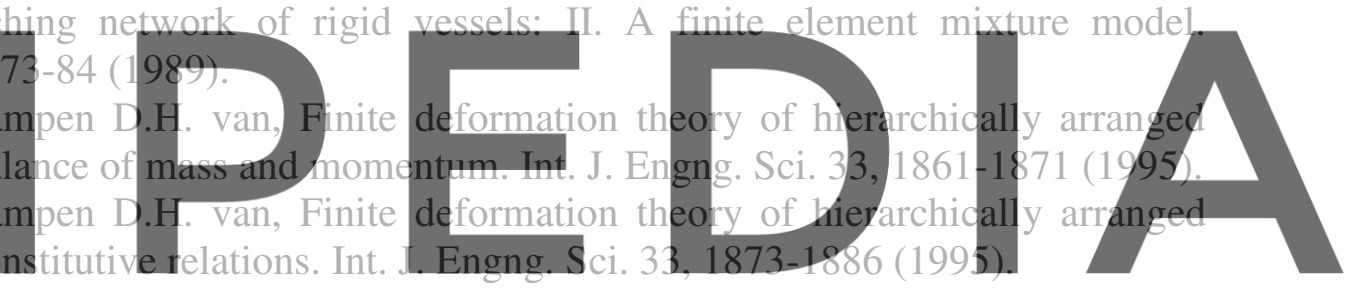

[17] Vankan W.J., Huyghe J.M., Janssen J.D., Huson A. A 3D finite element model of blood

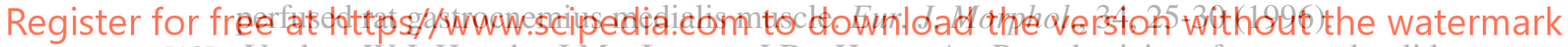

[18] Vankan W.J.,Huyghe J.M., Janssen J.D., Huson A., Poroelasticity of saturated solids with an application to blood perfusion. Int. J. Engng. Sci. 34, 1019-1031 (1996).

[19] Vankan W.J., Huyghe J.M., Janssen J.D., Huson A., Hacking W.J.G. and Schreiner W., Finite element analysis of blood flow through biological tissue . Int. J. Engng. Sci. 35 , 375-385 (1997).

[20] Vankan W.J., Huyghe J.M., Drost M.R., Janssen J.D., Huson A. A finite element mixture model for hierarchical porous media. Int. J. Num. Meth. Eng. 40 , 193-210 (1997)

[21] Vankan W.J., Huyghe J.M., Slaaf D.W., van Donkelaar C.C., Drost M.R., Janssen J.D., Huson A. Finite element simulation of blood perfusion in muscle tissue during compression and sustained contraction. Am. J. Physiol. 273, H1587-1594 (1997)

[22] Antonio F. Miguel, Toward an optimal design principle in symmetric and asymmetric tree flow networks, Journal of Theoretical Biology 389101-109 (2016)

[23] Adrian Bejan1and Sylvie Lorente, Constructal law of design and evolution: Physics, biology, technology, and society, J. Appl.Phys. 113, 151301-20 (2013).

[24] Antonio F. Miguel, A general model for optimal branching of fluidic networks, Physica A 512 665-674. (2018) 
[25] Yu C., Malakpoor K., and Huyghe J.M.. A full three-dimensional transient mixed hybrid finite element model for swelling hydrogels with strain dependent permeability. Soft Matter14(19): 3834-3848 (2018)

[26] Yu C., Malakpoor K. and Huyghe J.M.. A mixed hybrid finite element framework for the simulation of swelling ionized hydrogels. Computational Mechanics, 63(5), 835-852 (2019).

[27] Yu C., Huyghe J.M and Malakpoor K., 2D mixed hybrid FEM of Lanir model. Procedia IUTAM 12:93-104 (2015)

Figures

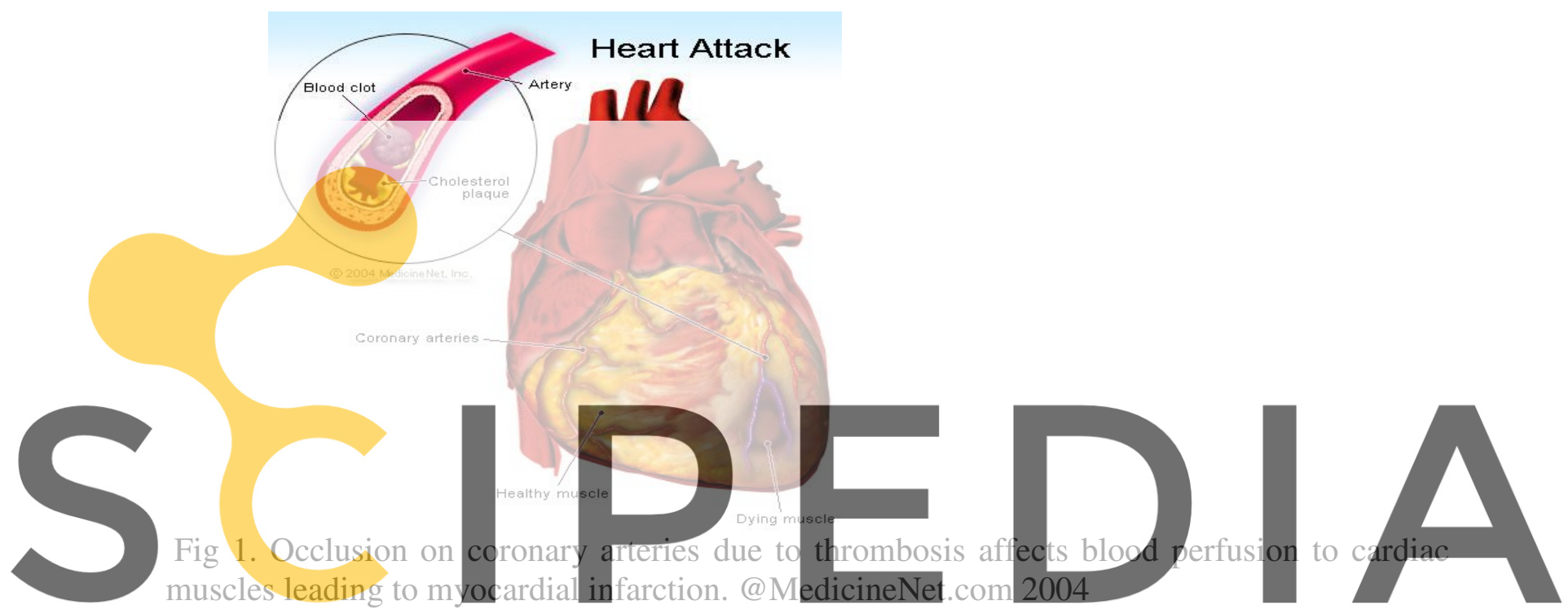




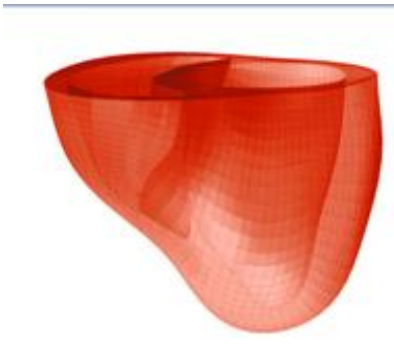

Undeformed mesh

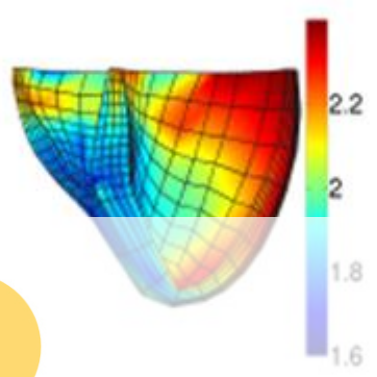

Sarcomere length $(\mu \mathrm{m})$

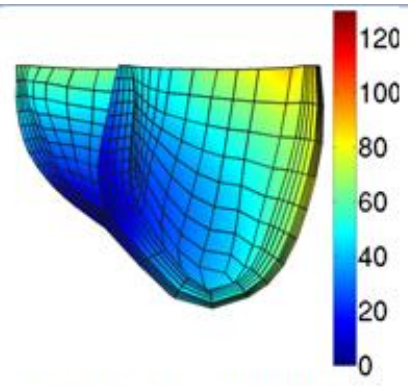

Electric depolarisation (mV)

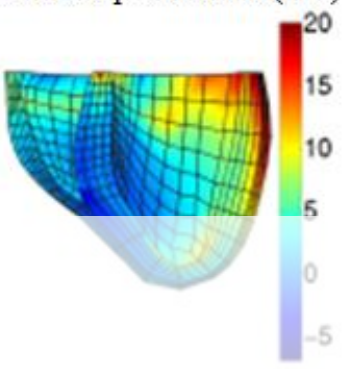

Stroke work $\left(\mathrm{mJ} / \mathrm{cm}^{3}\right)$

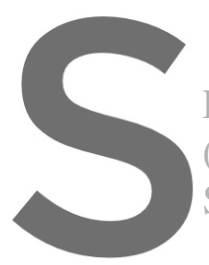

Fig. 2. The three-dimensional

(b) Electrical depolarization

Sarcomere length.[3]
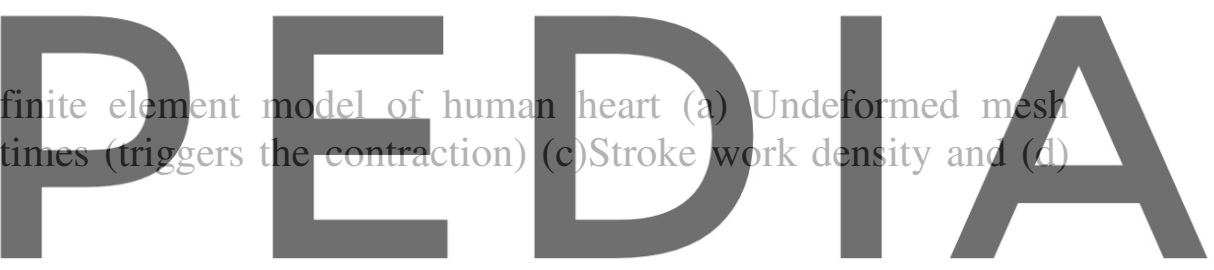

Register for free at https//www.scipedia.com to download the version without the watermark
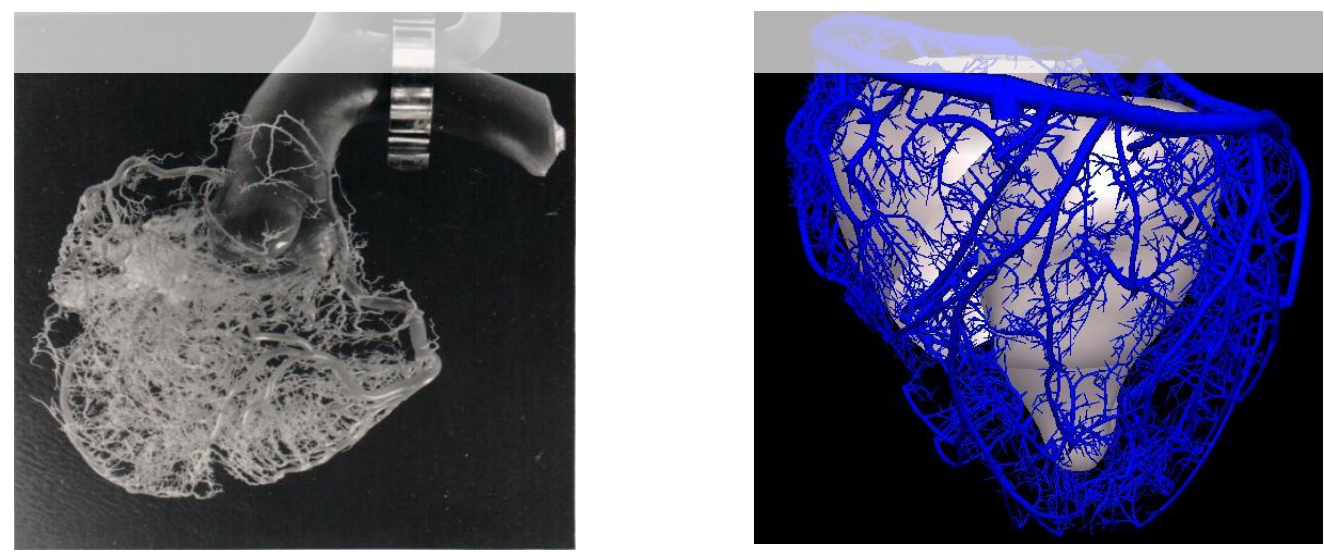

Fig. 3 (a) Polymer cast of the arterial coronary tree of a canine heart. (b) the left and right ventricles with white polymer. A tree of venous vessels mostly parallel to the arterial tree drains the blood with the blue vessels indicating the largest coronary veins. 


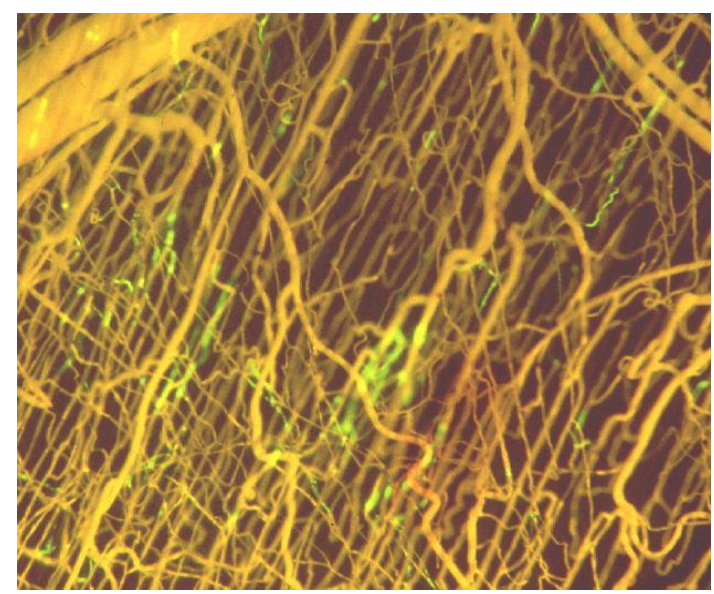

Fig. 4. Using low viscosity monomer, the smallest capillaries (5 micron in diameter) running parallel to the muscle fibers are visualised.

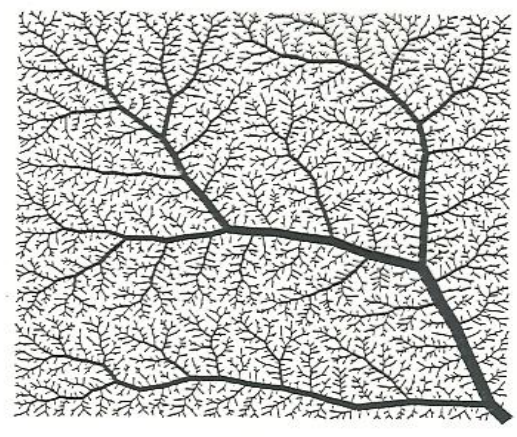

NW

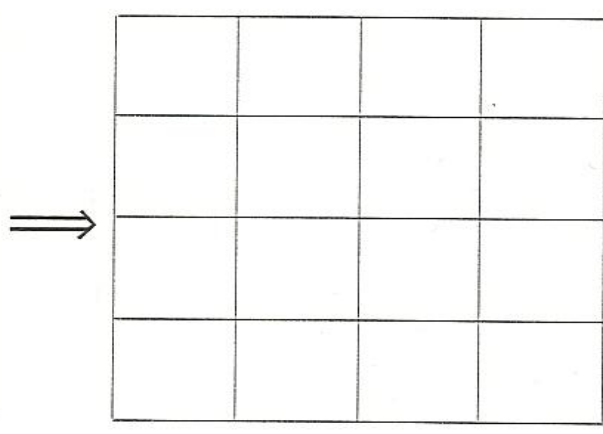

FE

Fig. 5. Pre-capillary pressures computed by means of network analysis (left) compare to continuum finite element analysis (right) 


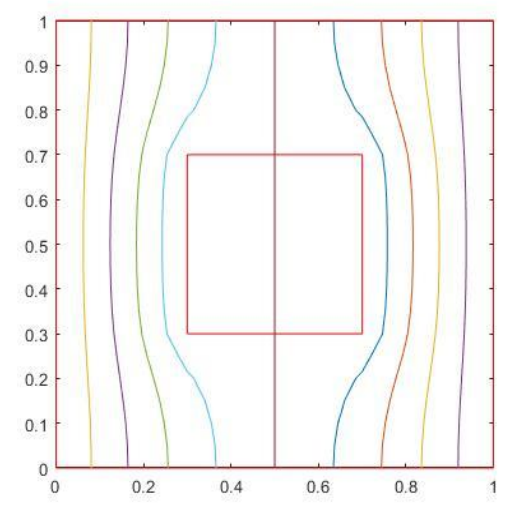

(a)

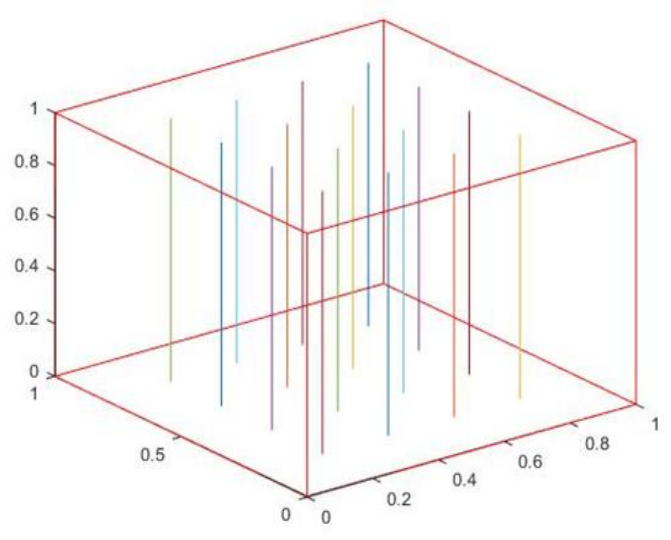

(b)

Fig. 6. Mixed hybrid finite element analysis for porous models in 2D and 3D. 